\title{
Group Management and Affective Dialogue in Collaborative Work
}

\begin{abstract}
Research and design of information systems in LIS has endeavoured to investigate human group tasks and the role of creativity. The current study builds on the premise behind Computer Supported Collaborative Work (CSCW), which is a design field aimed to facilitate group work through the application of computer technologies, driven by ever-changing contextual and environmental constraints. Using field observation, and a systematic quantification of the content of group conversation, the study formulates a description of the creative work of musicians, and the use of group management and affective dialogue during collaboration, with the goal of informing design. Results of the quantitative findings are presented with implications for future research.
\end{abstract}

\section{Background: Creative Collaboration and Group Management}

There is surprisingly little written in information science literature about the design of tools used to support the collaboration of creators, and in particular, the importance of dialogue used to facilitate group management. This pertains to ways in which group members involved in a common task use language to negotiate, manage, regulate and control social situations and collective states which are distinct from the task itself. Research in Library and Information Science strives to understand information behavior in different contexts with the goal of creating information systems which are aligned to real world needs. Research in Human Computer Interaction (HCI) similarly has looked for tools which may help facilitate creative work as a cognitive activity operating within environmental conditions (Pearce, \& Wiggins, 2002). Like music itself, the use of systems in music-making involves a sociocultural and theoretical approach which assesses behaviour with an emphasis on context (Hargreaves et al., 2012). Therefore, by applying reasoning based on contextual data such as group management interactions, tools provide ways to build domain knowledge by sharing of experiences, donating results, and motivating learners through expert feedback (Fischer, 2011).

Understanding collaboration through the use of language has been traditionally applied to work domains, but this method is also well-suited for informing the design collaborative systems to support creativity in non-work environments. The creative production process involves a wealth of data about social practices through which a creative work is conceived, negotiated, and codified, and are interactions that can be made explicit in order to provide a source of rich descriptions of the music itself. Burnard and Younker (2004) investigated how to help identify diversity in composing in the interplay of activities such as verification, incubation, preparation and exploration. Existing relationships between musicians is a factor of strong intuitive import to collaborative creation. Wiggins (2007) argues that there is a greater likelihood of transactive communication between groups of friends than those with no prior relationship, and that communication is affected by the existence of friendship. Appropriable systems should support the different perspectives that different people might have on information, implying a separation between information and the structures that describe it (Shah 2012). Research on creativity have led researchers to believe that creative art expression might play a major role in expression of their emotions, such as excitement, anger, or joy (Riley et al., 2009). Discoveries of this sort point to potential investigative area that the notion of "invisible work" is one which may also be 
explored through non-workplace settings for aesthetic and emotional realization. Open-ended and creative activities invariably involve tasks secondary to the creative process, namely, those which function to construct a shared social reality and maintain mutual engagement.

The current study addresses these issues by examining the use of group management dialogue involved in the authorship of a shared creative artefact. Findings reveal how group regulating feedback, mutual support, and expressed positive and negative affect can influence the process at critical moments in the collaboration to overcome blockages and reinforce successful alternatives. The compositionality of properties of objects in the collaboration allows members of a group to organize a common corpus for their different individual needs, while properties also act as points of coordination between them.

\section{Research Question}

How do collaborators use group management communication to affect the progress and outcomes of creative group authorship?

\section{Methods}

Rock Lottery is an invitation-only charity music performance held through an arts organization in London, Ontario, whereby 25-35 local rock musicians are assembled at random into distinct bands to compose and perform original music. The participants involved represent a crosssection of practicing, non-professional artists who perform in local bands. It was an ideal environment for interfacing with and observing musicians collaborating in a live musical enterprise. Members of Rock Lottery were chosen to participate so their composing sessions were recorded in the form of text transcripts. Data was gathered data over the course of 9 composing sessions lasting 2 hours each, involving 3 different musical groups.

The data analysis method, conversation analysis (Tan et al., 2014), was used to examine the minutiae of interaction at the conversational level. It identified the specific devices, such as conversational openings or adjacency pairs, that participants use to organize their talk. Characteristics such as turn-taking, speaker choice and speech act type were subsequently flagged and counted in the transcript. Examination of conversational transactions were used to specify how information is generated, shared and modified to overcome blockages as they arise. A blockage is defined by a momentary halt to collaboration due to some identified knowledge gap made apparent by the group. Conversational acts translate to the products of collaboration as clusters of codes warranting more detailed examination.

\section{Findings}

Four major types of group management dialogue were identified in the transcript and are summarized in Table 1 together with an example usage.

\begin{tabular}{|l|l|l|}
\hline Dialogue Type & Description & Example \\
\hline
\end{tabular}


CAIS Extended Abstract - January, 27, 2017

\begin{tabular}{|l|l|l|}
\hline Regulation & $\begin{array}{l}\text { Indicates regulation processes directed to } \\
\text { influence the partner's cognition, motivation or } \\
\text { action. }\end{array}$ & $\begin{array}{l}\text { We'll decide on song preference } \\
\text { order for when we're playing. Yeah, } \\
\text { it will be fun! }\end{array}$ \\
\hline Grounding & $\begin{array}{l}\text { Represents attempts to establish shared } \\
\text { understandings with partner through the use } \\
\text { of questions (e.g., clarifying what is meant by } \\
\text { the preceding statement; whether it be related } \\
\text { to the problem task, proposed solution, a } \\
\text { strategy, or a future activity). }\end{array}$ & $\begin{array}{l}\text { Drums: Did you come in on new } \\
\text { verse deliberately like that? It } \\
\text { sounded cool that way. }\end{array}$ \\
\hline Affective & $\begin{array}{l}\text { Expresses positive affect or emotion related to } \\
\text { task. It includes exclamations, humour, teasing, } \\
\text { thanks, apologies, empathy valuing partner's } \\
\text { perspective and contribution (e.g., referencing } \\
\text { others, acknowledgment, polite markers, } \\
\text { encouragement). }\end{array}$ & $\begin{array}{l}\text { Bass: We're going to sound like a } \\
\text { million bucks - we'll sound like iron } \\
\text { maiden up there! }\end{array}$ \\
\hline Disaffective & $\begin{array}{l}\text { Expresses negative affect or emotion, including } \\
\text { disengagement from the task. Altogether, } \\
\text { these instances demonstrate the group's } \\
\text { collective ability to collectively self-examine, } \\
\text { and to reflect on and repurpose group } \\
\text { objectives, strategies, processes and solutions. }\end{array}$ & $\begin{array}{l}\text { Lead: Yo, you're really going to have } \\
\text { to play something more forceful. It's } \\
\text { not sounding good like that at all. }\end{array}$ \\
\hline
\end{tabular}

Table 1: Management Dialogue Types

The frequency of each group management conversation type was averaged for all 3 groups across all 9, 2 hour composing sessions. The frequencies are summarized across time in Figure 1 . 


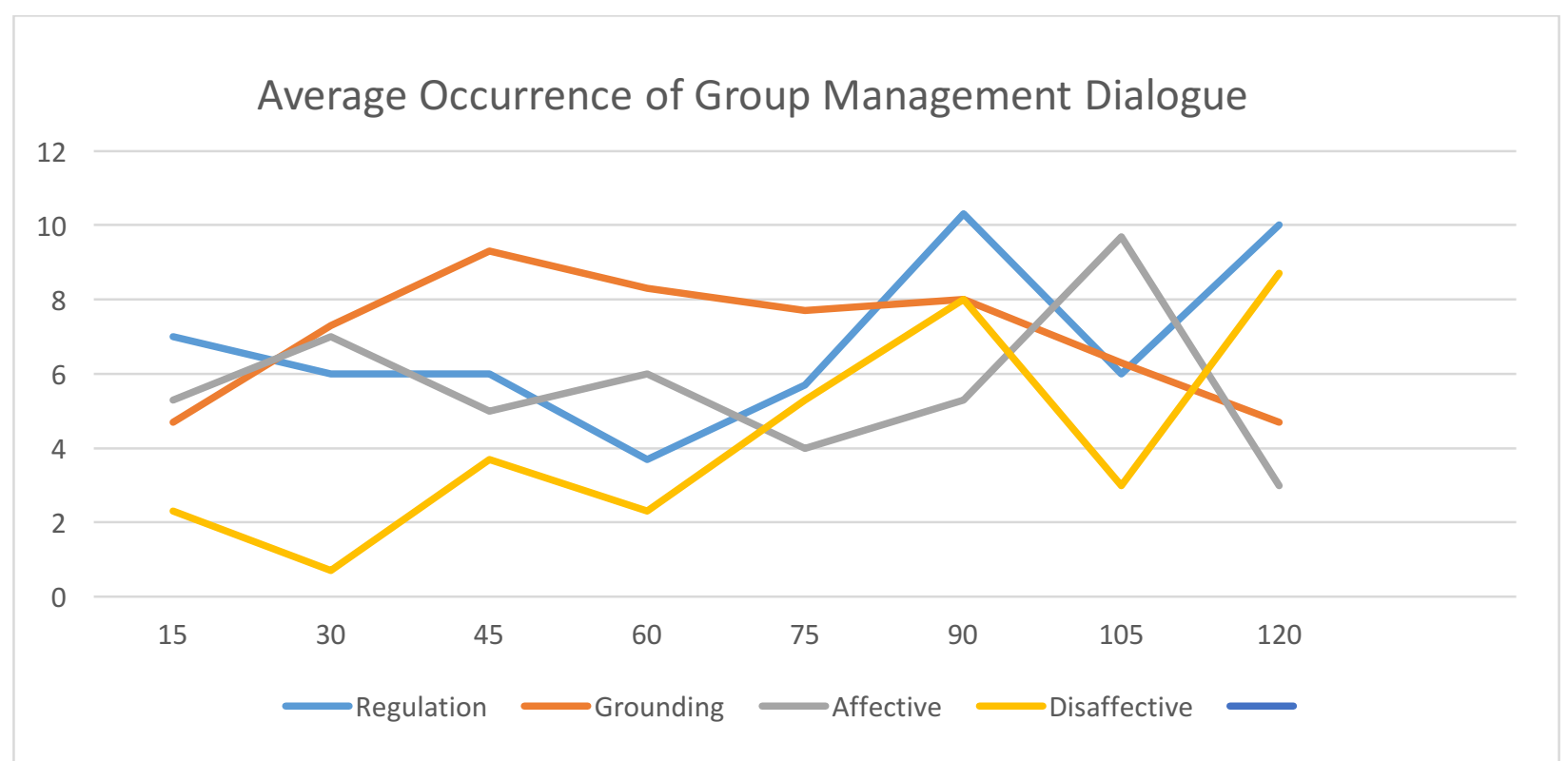

Figure 1: Average counts of dialogue across time, as lines of text, totaled over 3 sessions for all 3 musical groups

\section{Discussion}

Instances of management dialogue were instrumental in reinforcing existing ideas, and are distinguished from attempts to alter existing content or introduce new idea. Dialogic acts about management goals are prevalent at key points in the collaboration, translate to collaboration success. Data from conversational trends, indicate that divergent conversational patterns, including positive affect dialogic acts, spike at roughly halfway points in the collaboration timeline, which was common to all three sessions and groups. Subsequent transcript dialogue indicates evidence of positive knowledge construction and learning occurring at these moments. Maintaining states of positive affect was important throughout the collaboration, as new topics or situations which caused problematic group understanding were often alleviated through encouraging and optimistic reinforcement of previously established plans. Strategic use of affective and management language was found to be instrumental in bridging knowledge gaps and moving the collaboration forward. Dialogue moves that express positive team-directed affect or emotion are intrinsically functional to the task at hand, and demonstrates consulting and valuing the partner's perspective and contribution (e.g., referencing others, acknowledgment, polite markers, encouragement, addressing the group). Planning dialogic acts were supported with concentrated occurrences of a number of prosocial dialogue acts, namely, mutual grounding and cohesive-talk. This situation was also true for all three groups.

Affective properties which form the topical content of the collaboration translate to successful collaboration. The use of group management discourse, such as grounding questioning and cohesive talk - e.g., "that was awesome," "It sounds all right," "we're going to win!" — and similar social management use of language served to create positive encouragement for the group, and was helpful in establishing and justifying the context and priority of plans. Conversely, the occurrence of negative affect was not necessarily detrimental to constructive progress and served to motivate members to focus on the task at hand. Finally, a trend emerged in conversational references that cohesive talk and talking off topic are instrumental in supporting collaboration and are indicative of approval and resolution within the group. 


\section{Conclusion}

The study describes a way to apply quantitative observations to inform qualitative assessment of the characteristics of collaboration in groups. Using open-ended and creative activities to examine discourse secondary to the creative product, this study presents how group management communication serves groups to construct a shared reality and maintain mutual engagement. The prevalence of group regulating feedback, positive and negative affect and mutual grounding influences the process at critical junctures in the collaboration to overcome blockages. The data presented can inform the design of virtual systems used for collaboration, by making the progress of the creative product explicit at each stage of the collaboration, to support interaction that enhances mutual grounding, and to make visible affective expressions between participants to encourage reinforcement. In this way, design is based on naturalistic, real world, needs. For musicians, technical resources are viewed as the catalyst through which people manage these needs. Using an environment which forms a potential microcosm of virtual interaction between groups, it provides a framework for understanding and designing collaborative interaction in terms of dialogue features.

\section{References}

Burnard, P., \& Younker, B. (2004). Problem solving and creativity: Insights from students' individual composing pathways. International Journal of Music Education, 22(1), 5976.

Fischer, G. (2011). Social creativity: Exploiting the power of cultures of participation. In SKG2011: 7th International Conference on Semantics, Knowledge and Grids (pp. 18). Los Alamitos, CA: Conference Publishing Services.

Hargreaves, D., Meill, D., \& MacDonald, R. (2012). Musical imaginations: Multidisciplinary perspectives on creativity. London, UK: Oxford University Press.

Pearce, M., \& Wiggins, G. (2002). Aspects of a cognitive theory of creativity in musical composition. In Proceedings of 2nd International Workshop on Creative Systems, European Conference on Artificial Intelligence (pp. 17-24).

Riley, P., Alm, N., \& Newell, A. (2009). An interactive tool to promote musical creativity in people with dementia. Computers in Human Behaviour, 25, 599-608.

Tan, J., Caleon, I., Jonathan, C. \& Koh, E. (2014). A Dialogic framework for assessing collective creativity in computer-supported collaborative problem-solving tasks. Research and Practice in Technology Enhanced Learning, 9(3). 411- 437.

Wiggins, J. (2007). Compositional process in music. In L. Bresler (Ed.), International handbook of research in arts education (Vol. 16) (pp. 453-470). Dordrecht, NL: Springer. 
CAIS Extended Abstract - January, 27, 2017 